Correspondence

Elvira Garza-González

elvira_garza_gzz@yahoo.com

Received 6 June 2010

Accepted 1 October 2010

\section{Molecular characterization and antimicrobial susceptibility of extended-spectrum $\beta$-lactamase- producing Enterobacteriaceae isolates at a tertiary- care centre in Monterrey, Mexico}

\author{
Elvira Garza-González, ${ }^{1} \dagger$ Sandra Iveth Mendoza Ibarra, ${ }^{1} \dagger$ \\ Jorge M. Llaca-Díaz ${ }^{2}$ and Gloria M. Gonzalez ${ }^{1}$ \\ ${ }^{1}$ Departamento de Microbiología, Facultad de Medicina, Universidad Autónoma de Nuevo León, \\ Monterrey, Nuevo León, Mexico \\ ${ }^{2}$ Hospital Universitario 'Dr José Eleuterio González', Universidad Autónoma de Nuevo León, \\ Monterrey, Nuevo Leon, Mexico
}

\begin{abstract}
Our objective was to analyse phenotypic and genetic data of extended-spectrum $\beta$-lactamase (ESBL)-producing Klebsiella pneumoniae, Enterobacter cloacae, Escherichia coli and Serratia marcescens that cause infections in our hospital. Over a 3 year period, 342 randomly selected clinical Enterobacteriaceae isolates were tested for ESBL production and evaluated for the presence of the $\beta$-lactamase genes b/a $a_{\mathrm{SHV}}, b / a_{\mathrm{TEM}}, b / a_{\mathrm{CTX}-\mathrm{M}}$ and $b / a_{\mathrm{TLA}-1}$. The antibiotic susceptibilities of these isolates were also determined, and the clonality of the isolates was assessed by PFGE. Based on our analyses, 33/92 (35.9\%) K. pneumoniae, 31/87 (35.6\%) Enterobacter cloacae, 24/80 (30\%) E. coli and 17/83 (20.5\%) S. marcescens were identified as ESBL producers. The presence of TEM, SHV or CTX ESBL types was detected in 99/105 (94\%) of the isolates. TLA-1 was not detected in any of the 105 isolates. The dominant ESBL types were bla $_{\mathrm{SHV}-5}(n=33)$, bla $\mathrm{SHV}_{12}(n=31)$ and bla $\mathrm{CTX}_{\mathrm{M}-15}(n=30)$. The predominant ESBL identified in E. coli and Enterobacter cloacae isolates was CTX-M-15, whereas in K. pneumoniae and $S$. marcescens the predominant types were SHV-12 and SHV-5, respectively. PFGE genotyping revealed two main genetic patterns in the K. pneumoniae isolates, types SHV-12 and TEM-1 + SHV-5. An outbreak caused by Enterobacter cloacae SHV-5 + CTX-M-15 was detected. In contrast, most ESBL-producing isolates of $E$. coli and S. marcescens did not have similar PFGE banding patterns and thus were not genetically similar. Enterobacteriaceae are a concern in our hospital, especially K. pneumoniae and Enterobacter cloacae. Our results confirm that the CTX-M-15 ESBL type has spread rapidly in the hospital, and thus requires careful monitoring.
\end{abstract}

\section{INTRODUCTION}

Enterobacteriaceae rendered resistant to penicillins, broadspectrum cephalosporins and monobactams, by the production of extended-spectrum $\beta$-lactamases (ESBLs), are recognized as major causes of both nosocomial and community-acquired infections (Bradford, 2001; Cantón et al., 2008). ESBLs are found predominantly in Klebsiella species and Escherichia coli, but have been described in other species as well, including Enterobacter, Serratia, Citrobacter, Proteus and Salmonella (Paterson \& Bonomo, 2005).

†These authors contributed equally to this work.

Abbreviations: CSF, cerebrospinal fluid; ESBL, extended-spectrum $\beta$ lactamase.
The prevalence of ESBL-expressing bacteria varies across different geographical regions. Fewer than $10 \%$ of isolated strains express ESBLs in Sweden, Japan and Singapore, compared to rates higher than $30 \%$ in Portugal, Italy, New York and some Latin American countries. In Turkey, as many as $58 \%$ of isolated strains express ESBLs (Paterson \& Bonomo, 2005).

ESBLs arise mainly due to mutations in $\beta$-lactamases encoded by the $b l a_{\mathrm{SHV}}, b l a_{\mathrm{TEM}}$, and $b l a_{\mathrm{CTX}-\mathrm{M}}$ genes. At present, more than 300 different ESBL variants have been described (Paterson \& Bonomo, 2005). Though TEM and SHV variants are the most common ESBLs, during the past decade strains expressing CTX-M ESBLs have begun to emerge in many countries (Bonnet, 2004) and are now the most frequent non-TEM, non-SHV ESBL type. CTX-M 
$\beta$-lactamases are characterized by selective hydrolysis of cefotaxime rather than ceftazidime, though some CTX-M types, such as CTX-M-15, may actually hydrolyse ceftazidime (Poirel et al., 2001). Other ESBL enzymes, such as OXA, VEB, PER and GES, are less frequently encountered (Paterson \& Bonomo, 2005). In Mexico, several studies have reported the high prevalence of SHV-5 (Espinosa de los Monteros et al., 2008; Miranda et al., 2004). A novel ESBL type, TLA-1 (Silva et al., 2000), has been associated with SHV-5 (Alcantar-Curiel et al., 2004). In this report, we present a comprehensive phenotypic and genetic analysis of ESBL-producing infectious Klebsiella pneumoniae, Enterobacter cloacae, E. coli and Serratia marcescens isolated from a tertiary care hospital in Mexico.

\section{METHODS}

Clinical isolates. A total of 342 randomly selected $K$. pneumoniae $(n=92)$, E. coli $(n=80)$, Enterobacter cloacae $(n=87)$ and S. marcescens $(n=83)$ isolates were collected from the tertiary-care Hospital Universitario 'Dr José Eleuterio González' in Monterrey, Mexico, from January 2006 to January 2009. Information was collected about the patients from whom strains were isolated, including age, sex and the hospital unit where the patient stayed. Clinical specimens included those from the lower respiratory tract $(n=138)$, wounds $(n=43)$, blood $(n=38)$, fluids other than cerebrospinal fluid (CSF) and blood $(n=34)$, abscesses $(n=32)$, catheters $(n=30)$, CSF $(n=9)$ and urine $(n=18)$. Only one isolate per patient was included in our analyses.

Clinical isolates were identified using standard biochemical methods (Murray et al., 2007). When needed, API 20E galleries were used according to the manufacturer's instructions, and results were interpreted using API $20 \mathrm{E}$ V4.1 identification software. Bacterial isolates were stored in Brucella broth containing $15 \%$ glycerol at $-70{ }^{\circ} \mathrm{C}$.

ESBL production and characterization. The ESBL phenotypic detection test was performed using the combination disc method, using ceftazidime, cefotaxime and the inhibitory effect of clavulanic acid recommended by the Clinical and Laboratory Standards Institute (CLSI, 2009). When an ESBL phenotype was detected, PCR assays were conducted to test isolates for the presence of the $b l a_{\mathrm{SHV}}, b l a_{\mathrm{TEM}}$, $b l a_{\mathrm{CTX}-\mathrm{M}}$ and $b l a_{\mathrm{TLA}-1}$ genes. To obtain template DNA, 3-5 colonies from each isolate were boiled in sterile water for $5 \mathrm{~min}$. Samples were centrifuged and $2 \mu$ supernatant was used as the DNA template. The PCRs were carried out using published primers (Berçot et al., 2010; Coque et al., 2002; Siu et al., 1999). PCR products were purified using the Wizard PCR preps DNA purification system (Promega). All bidirectional nucleotide sequencing was performed at the Instituto de Biotecnología, Universidad Nacional Autónoma de México (Cuernavaca, Morelos, Mexico), using the above PCR primers. Nucleotide sequences and the derived amino acid sequences were compared with previously described sequences (GenBank database http://www.ncbi.nlm.nih.gov/ and www.lahey.org/studies, respectively).

Antibiotic susceptibility testing was performed on all ESBL producers using the broth microdilution method as described by the CLSI (2009). Six $\beta$-lactam antibiotics were tested: ceftriaxone, ceftazidime, cefepime, ampicillin, piperacillin and meropenem. Isolates were also tested against non- $\beta$-lactam antibiotics, including amikacin, levofloxacin and tigecycline.

PFGE. Isolates were genotyped using PFGE. Genomic DNA from each strain was isolated in an agarose-embedded form and subjected to in-gel enzymic digestion using $50 \mathrm{U} \mathrm{XbaI}$. The agarose plug was subsequently placed in the preformed wells of an agarose gel. PFGE was performed for $23 \mathrm{~h}$ with a CHEF Mapper system (Bio-Rad) using $0.5 \times$ TBE buffer $(10 \times$ TBE: $890 \mathrm{mM}$ Tris-borate, $890 \mathrm{mM}$ boric acid, $20 \mathrm{mM}$ EDTA). PFGE profiles were visualized under UV after staining with ethidium bromide $\left(0.5 \mathrm{mg} \mathrm{ml}^{-1}\right)$. Patterns were interpreted using the Tenover criteria (Tenover et al., 1995).

\section{RESULTS AND DISCUSSION}

\section{ESBL production and resistance rates}

Using the phenotypic assay, 33/92 (35.9\%) of $K$. pneumoniae, 31/87 (35.6\%) of Enterobacter cloacae, 24/80 (30\%) of E. coli and 17/83 (20.5\%) of S. marcescens were identified as ESBL producers. All of the ESBL producers tested positive for at least one of the $\beta$-lactamase encoding genes by PCR. However, ESBLs could be sequenced in only 99 of the 105 isolates. The six other isolates contained $b l a_{\text {TEM-1 }}$, which is not an ESBL (Table 1). The dominant ESBL types detected were $b l a_{\mathrm{SHV}-5} \quad(n=33), \quad b l a_{\mathrm{SHV} 12}$ $(n=31)$ and $b l a_{\text {CTX-M-15 }}(n=30)$.

A particularly interesting result was the finding that $66.7 \%$ of the E. coli and $38.7 \%$ of the Enterobacter cloacae isolates contained CTX-M-15. In contrast to other CTX-M types, this ESBL type may hydrolyse ceftazidime (Poirel et al., 2001). CTX-M-15 has been detected in Salmonella sp. (Rotimi et al., 2008) and is believed to be the dominant ESBL type in some Asian countries (Bonnet, 2004), occurring in $99.6 \%$ of E. coli and $99.2 \%$ of $K$. pneumoniae isolates in Thailand (Kiratisin et al., 2008). The only report of the CTX-M-15 type in Mexico was made recently in $E$. coli (Rocha-Gracia et al., 2010). Our study confirms that the CTX-M-15 ESBL has spread in Enterobacteriaceae, because it was the most prevalent ESBL in the E. coli and Enterobacter cloacae isolates analysed in this study.

To our knowledge, CTX-M ESBLs are not the predominant type in Mexican hospitals. SHV ESBLs, along with a new ESBL named TLA-1, are more commonly reported in these institutions (Espinosa de los Monteros et al., 2008; GarzaRamos et al., 2009; Silva et al., 2000). None of the isolates in this study contained TLA-1.

The dominant ESBL in K. pneumoniae was SHV-12 (51.5\%) and in S. marcescens it was SHV-5 (47.1\%). The isolation of S. marcescens SHV-5 is somewhat relevant because it represents a serious threat in the hospital, as was shown by a previous outbreak caused by a cephalosporin-resistant $S$. marcescens SHV-5 producing strain reported in a Mexican hospital. In this outbreak, the strain was isolated from patients, from medical staff and from chlorhexidine disinfectant solutions (Espinosa de los Monteros et al., 2008).

High resistance rates were observed for levofloxacin and, as expected, for ampicillin. In contrast, low resistance was observed for amikacin and tigecycline, and none of the strains was resistant to meropenem. The most highly resistant ESBL strains were Enterobacter cloacae SHV5+ CTX-M-15, Enterobacter cloacae TEM-1 + CTX-M-15, 
Table 1. Distribution of the bla $a_{\mathrm{TEM}}$, bla $a_{\mathrm{SHV}}$ and bla $\mathrm{C}_{\mathrm{CTX}} \mathrm{ESBLs}$ and antimicrobial susceptibility

All isolates had $0 \%$ resistance to meropenem.

\begin{tabular}{|c|c|c|c|c|c|c|c|c|c|}
\hline \multirow[t]{2}{*}{ Species and ESBL type ${ }^{\star}$} & \multirow{2}{*}{$\begin{array}{c}\text { No of } \\
\text { isolates }(\%)\end{array}$} & \multicolumn{8}{|c|}{ Percentage resistant to: } \\
\hline & & AMK & LVX & CRO & CAZ & PIP & FEP & AM & TIG \\
\hline \multicolumn{10}{|l|}{ Enterobacter cloacae $(n=31)$} \\
\hline TEM-1 + SHV-12 & $8(7.6)$ & 25 & 13 & 50 & 75 & 13 & 13 & 13 & 13 \\
\hline TEM-1 + CTX-M15 & $6(5.7)$ & 17 & 50 & 100 & 83 & 0 & 67 & 100 & 0 \\
\hline SHV-5 + CTX-M15 & $5(4.8)$ & 100 & 20 & 100 & 60 & 20 & 20 & 100 & 0 \\
\hline TEM-1 & $4(3.8)$ & 0 & 25 & 50 & 100 & 25 & 25 & 100 & 25 \\
\hline TEM-1 + SHV-5 & $4(3.8)$ & 25 & 0 & 75 & 75 & 50 & 50 & 100 & 0 \\
\hline SHV-2 & $2(1.9)$ & 0 & 0 & 0 & 50 & 0 & 0 & 100 & 0 \\
\hline TEM-1 + SHV-5 + CTX-M15 & $1(1)$ & 0 & 0 & 100 & 0 & 0 & 0 & 100 & 0 \\
\hline TEM-1 + SHV-2 & $1(1)$ & 0 & 100 & 0 & 100 & 0 & 0 & 100 & 0 \\
\hline \multicolumn{10}{|l|}{ Escherichia coli $(n=24)$} \\
\hline TEM-1 + CTX-M15 & $11(10.5)$ & 9 & 100 & 100 & 27 & 0 & 73 & 100 & 0 \\
\hline CTX-M15 & $5(4.8)$ & 0 & 100 & 20 & 60 & 20 & 40 & 100 & 20 \\
\hline SHV-2 & $2(1.9)$ & 0 & 100 & 100 & 50 & 0 & 50 & 100 & 0 \\
\hline TEM-1 + SHV-5 & $2(1.9)$ & 0 & 50 & 50 & 0 & 0 & 0 & 100 & 0 \\
\hline SHV-5 & $2(1.9)$ & 0 & 100 & 100 & 0 & 0 & 50 & 100 & 0 \\
\hline TEM-1 + SHV-12 & $1(1)$ & 0 & 100 & 0 & 0 & 0 & 0 & 100 & 0 \\
\hline TEM-1 & $1(1)$ & 0 & 100 & 0 & 0 & 0 & 0 & 100 & 0 \\
\hline \multicolumn{10}{|l|}{ Klebsiella pneumoniae $(n=33)$} \\
\hline SHV-12 & $17(16.2)$ & 35 & 76 & 53 & 82 & 35 & 24 & 94 & 6 \\
\hline TEM-1 + SHV-5 & $11(10.5)$ & 27 & 27 & 55 & 73 & 18 & 9 & 91 & 9 \\
\hline TEM-1 + CTX-M15 & $2(1.9)$ & 0 & 100 & 50 & 50 & 0 & 0 & 100 & 0 \\
\hline SHV-2 & $2(1.9)$ & 0 & 50 & 50 & 50 & 0 & 0 & 100 & 0 \\
\hline TEM-1 & $1(1)$ & 100 & 100 & 100 & 100 & 100 & 0 & 100 & 0 \\
\hline \multicolumn{10}{|l|}{ Serratia marcescens $(n=17)$} \\
\hline SHV-5 & $8(7.6)$ & 50 & 0 & 38 & 50 & 38 & 38 & 100 & 13 \\
\hline SHV-12 & $5(10.5)$ & 0 & 60 & 60 & 40 & 100 & 20 & 100 & 20 \\
\hline SHV-2 & $4(3.8)$ & 0 & 0 & 50 & 0 & 0 & 0 & 50 & 0 \\
\hline
\end{tabular}

AM, Ampicillin; AMK, amikacin; CAZ, ceftazidime; CRO, ceftriaxone; FEP, cefepime; LVX, levofloxacin; PIP, piperacillin; TIG, tigecycline. ${ }^{\star}$ TEM-1 is not an ESBL.

E. coli TEM-1 + CTX-M-15, K. pneumoniae SHV-12 and S. marcescens SHV-12. These strains were more than $50 \%$ resistant to four of the nine antibiotics tested. The high resistance rates observed for these isolates are not surprising, as bacteria that produce CTX-M-type $\beta$-lactamases typically have high cefotaxime MICs, of greater than $64 \mu \mathrm{g}$ $\mathrm{ml}^{-1}$. In particular, CTX-M-15 may actually hydrolyse ceftazidime, with an MIC as high as $256 \mu \mathrm{g} \mathrm{ml}^{-1}$ (Baraniak et al., 2002; Poirel et al., 2002). Additionally, cefepime MICs for the CTX-M ESBL types are greater than those observed for bacteria that produce other ESBL types (Yu et al., 2002). Furthermore, the SHV-12 type has been associated with high-level resistance to ceftazidime (Tansawai et al., 2009).

\section{Molecular epidemiology}

The majority of ESBL producers were isolated from lower respiratory tract specimens $(n=44)$, followed by catheter
( $n=15)$, blood $(n=14)$, wound $(n=11)$, fluids other than CSF and blood $(n=8)$, abscesses $(n=7)$, urine $(n=5)$ and CSF $(n=2)$. A pulsotype was defined as a group of isolates that displayed PFGE banding patterns $\geqslant 85 \%$ similar (Singh et al., 2006). Eighty-two pulsotypes could be described from the PFGE patterns of the isolates tested.

Our analyses revealed that $78.3 \%(18 / 23)$ of the typable $K$. pneumoniae isolates could be separated into two clones (Fig. 1, Table 2). A total of $37 \%$ (10/27) of Enterobacter cloacae isolates could be grouped into two clones, of which one clone (clone 3) was an SHV-5 + CTX-M-15 producer. Though SHV-5-producing Enterobacter cloacae strains have been described in Mexico (Garza-Ramos et al., 2009), this is, to the best of our knowledge, the first report of an Enterobacter cloacae strain isolated in Mexico that produces both SHV-5 and CTX-M15.

PFGE analysis revealed a high genomic heterogeneity among ESBL-producing E. coli and S. marcescens isolates. 


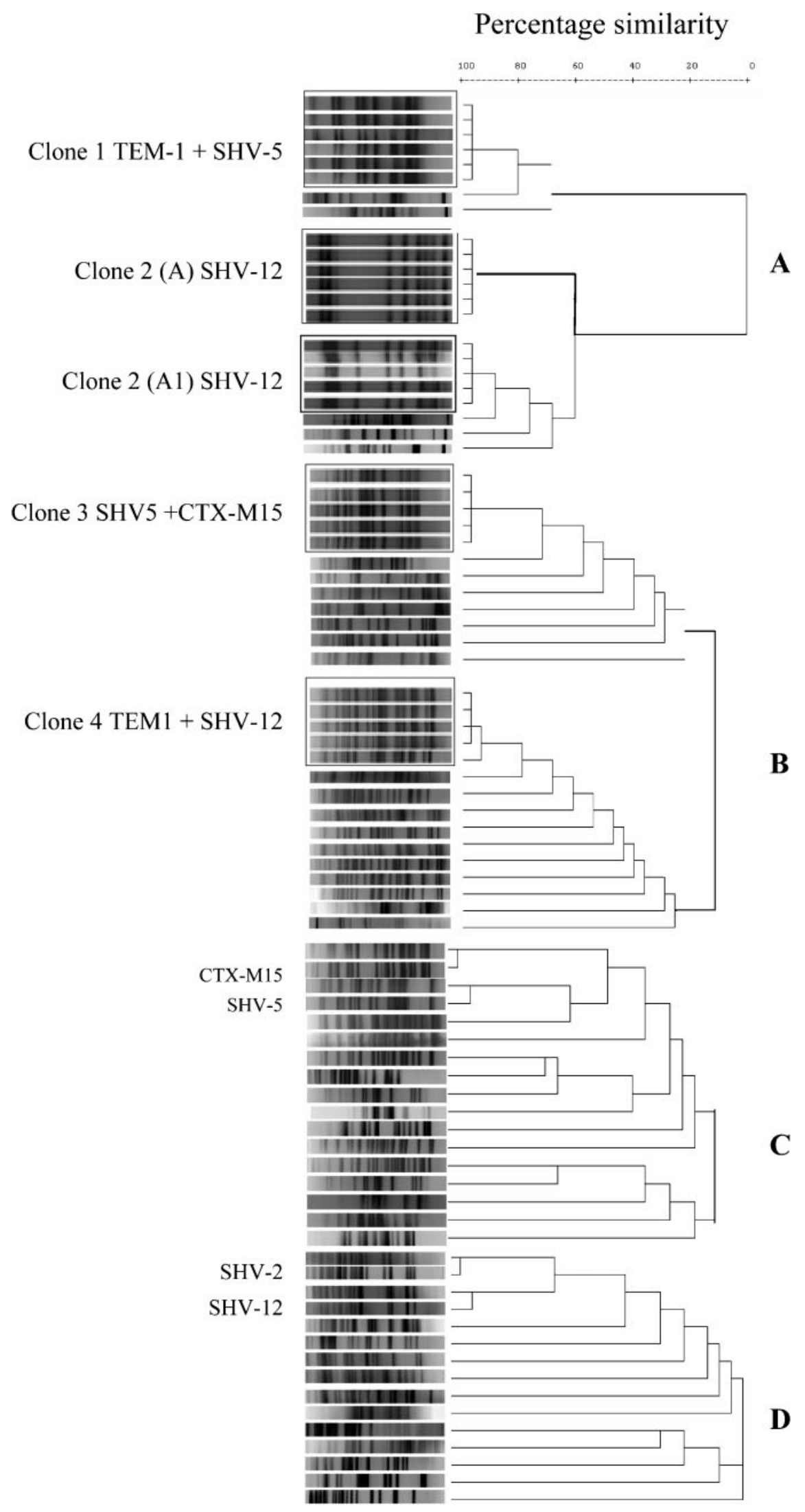

Fig. 1. PFGE analysis of K. pneumoniae (A), Enterobacter cloacae (B), E. coli (C) and S. marcescens (D) isolates. The boxes indicate an $85 \%$ banding pattern similarity, the standard used to define the clones. 
Table 2. ESBL type and clonal origin

Lower respiratory tract specimens, $85 \%$ mainly bronchial lavage.

\begin{tabular}{|c|c|c|c|c|c|c|c|}
\hline \multirow{2}{*}{$\begin{array}{l}\text { Isolate and ESBL } \\
\text { type }\end{array}$} & \multirow[t]{2}{*}{ Clone } & \multirow[t]{2}{*}{ Isolate } & \multirow[t]{2}{*}{ Specimen } & \multirow[t]{2}{*}{ Isolation date } & \multicolumn{3}{|c|}{ Patient } \\
\hline & & & & & Age (years) & Gender & Hospital ward \\
\hline \multirow{6}{*}{$\begin{array}{l}\text { Klebsiella } \\
\text { pneumoniae } \\
\text { TEM-1 + SHV-5 }\end{array}$} & 1 & 1146 & Fluids & $19 / 07 / 2006$ & 0 & M & Paediatrics \\
\hline & & 1503 & Blood & $29 / 08 / 2006$ & 0 & M & N-ICU \\
\hline & & 1504 & Catheter & $01 / 08 / 2006$ & NA & $\mathrm{M}$ & IM \\
\hline & & 1517 & Blood & $14 / 09 / 2006$ & 18 & M & IM \\
\hline & & 1716 & Blood & $14 / 09 / 2006$ & 18 & M & IM \\
\hline & & 4837 & Blood & $08 / 08 / 2007$ & 1 & $\mathrm{~F}$ & Paediatrics \\
\hline \multirow{14}{*}{$\begin{array}{l}\text { Klebsiella } \\
\text { pneumoniae } \\
\text { SHV12 }\end{array}$} & $2(\mathrm{~A})$ & 1262 & Catheter & $27 / 07 / 2006$ & 64 & M & IM \\
\hline & & 1715 & LR & $14 / 09 / 2006$ & 19 & $\mathrm{~F}$ & A-ICU \\
\hline & & 1717 & Catheter & $14 / 09 / 2006$ & 52 & $\mathrm{~F}$ & PS-ICU \\
\hline & & 1783 & Catheter & $20 / 09 / 2006$ & 38 & $\mathrm{~F}$ & PS-ICU \\
\hline & & 2805 & LR & $24 / 11 / 2006$ & 32 & $\mathrm{~F}$ & Surgery \\
\hline & & 4529 & Blood & $13 / 07 / 2007$ & NA & M & PS-ICU \\
\hline & & 4563 & LR & $18 / 07 / 2007$ & 65 & $\mathrm{~F}$ & Pensioner \\
\hline & & 5706 & Blood & $24 / 10 / 2007$ & 18 & $\mathrm{~F}$ & $\mathrm{IM}$ \\
\hline & $2(\mathrm{~A} 1)$ & 1753 & LR & $18 / 09 / 2006$ & 52 & $\mathrm{~F}$ & A-ICU \\
\hline & & 2406 & Blood & $23 / 10 / 2006$ & 16 & $\mathrm{~F}$ & Obstetrics \\
\hline & & 3444 & Blood & $06 / 03 / 2007$ & 48 & M & IM \\
\hline & & 5978 & LR & $27 / 11 / 2007$ & 24 & $\mathrm{~F}$ & A-ICU \\
\hline & & 7008 & LR & $20 / 05 / 2008$ & 72 & $\mathrm{~F}$ & A-ICU \\
\hline & & 7078 & LR & $23 / 05 / 2008$ & 25 & M & A-ICU \\
\hline \multirow{4}{*}{$\begin{array}{l}\text { Enterobacter } \\
\text { cloacae SHV- } \\
5+\text { CTX-M-15 }\end{array}$} & 3 & 5555 & LR & $04 / 10 / 2007$ & 64 & M & A-ICU \\
\hline & & 5602 & LR & $11 / 10 / 2007$ & 40 & M & A-ICU \\
\hline & & 5618 & LR & $12 / 10 / 2007$ & 42 & $\mathrm{~F}$ & A-ICU \\
\hline & & 6024 & LR & $04 / 12 / 2007$ & 86 & M & A-ICU \\
\hline \multirow{5}{*}{$\begin{array}{l}\text { Enterobacter } \\
\text { cloacae TEM- } \\
1+\text { SHV-12 }\end{array}$} & 4 & 540 & Fluids & $08 / 06 / 2006$ & 0 & $\mathrm{~F}$ & N-ICU \\
\hline & & 541 & Fluids & $08 / 06 / 2006$ & 70 & $\mathrm{M}$ & Pensioner \\
\hline & & 542 & Catheter & 08/06/2006 & 15 & M & Paediatrics \\
\hline & & 1050 & Blood & $10 / 07 / 2006$ & 0 & $\mathrm{~F}$ & N-ICU \\
\hline & & 1151 & LR & $19 / 07 / 2006$ & 19 & $\mathrm{~F}$ & A-ICU \\
\hline
\end{tabular}

A, Adult; F, female; ICU, Intensive Care Unit; IM, Internal Medicine; LR, lower respiratory tract specimens; M, male; N, neonate; NA, not available; PS, post surgery.

More than $70 \%$ of these isolates could not be assigned to clonal groups. This is in contrast to published reports that describe a high degree of genetic relatedness among E. coli and S. marcescens strains isolated in other hospitals (Paterson \& Bonomo, 2005).

In this study, we detected six isolates that contained TEM1 , which has been reported in many countries (Al-Obeid et al., 2008; Severin et al., 2010). However, TEM-1 is not an ESBL, and surveillance of strains carrying the TEM-1 $\beta$-lactamase is needed, as these strains may give rise to new ESBL types. Evidence that these strains can develop into new ESBLs was presented after an outbreak of a TEM-1containing Klebsiella oxytoca strain. After patients were treated with ceftazidime, $K$. oxytoca TEM-12 was detected in the same neonatal unit (Du Bois et al., 1995). In our study, TEM-1 was detected in K. pneumoniae, E. coli and Enterobacter cloacae (six isolates in total), which may increase the potential health risk of this non-ESBL type. In these isolates, the presence of other non-common ESBL 
types, such as OXA, VEB, PER and GES, may be responsible for the ESBL phenotype observed for the isolates characterized in this study, though additional testing to detect the presence of genes encoding the less common ESBL types is needed to confirm this.

In conclusion, K. pneumoniae and Enterobacter cloacae were the species with the highest ESBL production, and multiple CTX-M-15 isolates were detected in this Mexican hospital. The results of this study describe the genetic characteristics and molecular epidemiology of ESBLs among Enterobacteriaceae isolates at a tertiary care hospital in Mexico and provide insight into the emergence of bacterial strains harbouring ESBL genes.

\section{ACKNOWLEDGEMENTS}

We thank Maria de la Luz Acevedo and Carlos Paz for their technical assistance, and Dr Sergio Lozano for reviewing the manuscript. This work was supported by the Consejo Nacional de Ciencia y Tecnología (CONACyT) (grant no. 58591).

\section{REFERENCES}

Alcantar-Curiel, D., Tinoco, J., Gayosso, C., Carlos, A., Daza, C., Perez-Prado, M., Salcido, L., Santos, J. \& Alpuche-Aranda, C. (2004). Nosocomial bacteremia and urinary tract infections caused by extended-spectrum $\beta$-lactamase-producing Klebsiella pneumoniae with plasmids carrying both SHV-5 and TLA-1 genes. Clin Infect Dis 38, 1067-1074.

Al-Obeid, S., Bremont, S., Jabri, L., Massoudi, N. \& Haddad, Q. (2008). Klebsiella pneumoniae LO10 producing extended-spectrum $\beta$ lactamase SHV-12 in Saudi Arabia. J Chemother 20, 709-713.

Baraniak, A., Fiett, J., Hryniewicz, W., Nordmann, P. \& Gniadkowski, M. (2002). Ceftazidime-hydrolysing CTX-M-15 extended-spectrum $\beta$ lactamase (ESBL) in Poland. J Antimicrob Chemother 50, 393-396.

Berçot, B., Poirel, L., Silva-Sanchez, J. \& Nordmann, P. (2010). Association of the extended-spectrum $\beta$-lactamase gene $b a_{\mathrm{TLA}-1}$ with a novel ISCR element, ISCR20. Antimicrob Agents Chemother 54, 4026-4028.

Bonnet, R. (2004). Growing group of extended-spectrum $\beta$-lactamases: the CTX-M enzymes. Antimicrob Agents Chemother 48, 1-14

Bradford, P. A. (2001). Extended-spectrum $\beta$-lactamases in the 21 st century: characterization, epidemiology, and detection of this important resistance threat. Clin Microbiol Rev 14, 933-951.

Cantón, R., Novais, A., Valverde, A., Machado, E., Peixe, L., Baquero, F. \& Coque, T. (2008). Prevalence and spread of extended-spectrum $\beta$ lactamase-producing Enterobacteriaceae in Europe. Clin Microbiol Infect 14 (Suppl. 1), 144-153.

CLSI (2009). Performance Standards for Antimicrobial Susceptibility Testing, 19th informational supplement, M100-S19. Wayne, PA: Clinical and Laboratory Standards Institute.

Coque, T. M., Oliver, A., Pérez-Díaz, J. C., Baquero, F. \& Cantón, R. (2002). Genes encoding TEM-4, SHV-2, and CTX-M-10 extendedspectrum $\beta$-lactamases are carried by multiple Klebsiella pneumoniae clones in a single hospital (Madrid, 1989 to 2000). Antimicrob Agents Chemother 46, 500-510.

Du Bois, S. K., Marriott, M. S. \& Amyes, S. G. (1995). TEM- and SHVderived extended-spectrum $\beta$-lactamases: relationship between selection, structure and function. J Antimicrob Chemother 35, 7-22.
Espinosa de los Monteros, L. E., Silva-Sanchez, J., Jiménez, L. V., Rojas, T., Garza-Ramos, U. \& Valverde, V. (2008). Outbreak of infection by extended-spectrum $\beta$-lactamase SHV-5-producing Serratia marcescens in a Mexican hospital. J Chemother 20, 586592.

Garza-Ramos, U., Davila, G., Gonzalez, V., Alpuche-Aranda, C., López-Collada, V., Alcantar-Curiel, D., Newton, O. \& Silva-Sanchez, J. (2009). The $b l a_{\mathrm{SHV}-5}$ gene is encoded in a compound transposon duplicated in tandem in Enterobacter cloacae. Clin Microbiol Infect 15, 878-880.

Kiratisin, P., Apisarnthanarak, A., Laesripa, C. \& Saifon, P. (2008). Molecular characterization and epidemiology of extended-spectrum$\beta$-lactamase-producing Escherichia coli and Klebsiella pneumoniae isolates causing health care-associated infection in Thailand, where the CTX-M family is endemic. Antimicrob Agents Chemother 52, 2818-2824.

Miranda, G., Castro, N., Leaños, B., Valenzuela, A., Garza-Ramos, U., Rojas, T., Solórzano, F., Chihu, L. \& Silva, J. (2004). Clonal and horizontal dissemination of Klebsiella pneumoniae expressing SHV-5 extended-spectrum $\beta$-lactamase in a Mexican pediatric hospital. J Clin Microbiol 42, 30-35.

Murray, P. R., Baron, J. L., Jorgensen, J. H., Landry, M. L. \& Pfaller, M. A. (2007). Manual of Clinical Microbiology, 9th edn, vol. 1. Washington, DC: American Society for Microbiology.

Paterson, D. L. \& Bonomo, R. A. (2005). Extended-spectrum $\beta$ lactamases: a clinical update. Clin Microbiol Rev 18, 657-686.

Poirel, L., Naas, T., Le Thomas, I., Karim, A., Bingen, E. \& Nordmann, P. (2001). CTX-M-type extended-spectrum $\beta$-lactamase that hydrolyzes ceftazidime through a single amino acid substitution in the omega loop. Antimicrob Agents Chemother 45, 3355-3361.

Poirel, L., Gniadkowski, M. \& Nordmann, P. (2002). Biochemical analysis of the ceftazidime-hydrolysing extended-spectrum $\beta$-lactamase CTX-M-15 and of its structurally related $\beta$-lactamase CTX-M-3. J Antimicrob Chemother 50, 1031-1034.

Rocha-Gracia, R., Ruiz, E., Romero-Romero, S., Lozano-Zarain, P., Somalo, S., Palacios-Hernández, J., Caballero-Torres, P. \& Torres, C. (2010). Detection of the plasmid-borne quinolone resistance determinant qepA1 in a CTX-M-15-producing Escherichia coli strain from Mexico. J Antimicrob Chemother 65, 169-171.

Rotimi, V. O., Jamal, W., Pal, T., Sovenned, A. \& Albert, M. J. (2008). Emergence of CTX-M-15 type extended-spectrum $\beta$-lactamaseproducing Salmonella spp. in Kuwait and the United Arab Emirates. J Med Microbiol 57, 881-886.

Severin, J. A., Mertaniasih, N. M., Kuntaman, K., Lestari, E. S., Purwanta, M., Lemmens-Den Toom, N., Duerink, D. O., Hadi, U., van Belkum, A. \& other authors (2010). Molecular characterization of extended-spectrum $\beta$-lactamases in clinical Escherichia coli and Klebsiella pneumoniae isolates from Surabaya, Indonesia. J Antimicrob Chemother 65, 465-469.

Silva, J., Aguilar, C., Ayala, G., Estrada, M., Garza-Ramos, U., LaraLemus, R. \& Ledezma, L. (2000). TLA-1: a new plasmid-mediated extended-spectrum $\beta$-lactamase from Escherichia coli. Antimicrob Agents Chemother 44, 997-1003.

Singh, A., Goering, R., Simjee, S., Foley, S. \& Zervos, M. (2006). Application of molecular techniques to the study of hospital infection. Clin Microbiol Rev 19, 512-530.

Siu, L. K., Lu, P.-L., Hsueh, P.-R., Lin, F. M., Chang, S.-C., Luh, K.-T., Ho, M. \& Lee, C.-Y. (1999). Bacteremia due to extended-spectrum $\beta$ lactamase-producing Escherichia coli and Klebsiella pneumoniae in a pediatric oncology ward: clinical features and identification of different plasmids carrying both SHV-5 and TEM-1 genes. J Clin Microbiol 37, 4020-4027. 
Tansawai, U., Boonkerd, N., Polwichai, P., Dejsirilert, S. \& Niumsup, P. (2009). SHV-12 extended spectrum $\beta$-lactamase associated with highlevel ceftazidime resistance in Enterobacter cloacae isolated from Thailand. Southeast Asian J Trop Med Public Health 40, 148-154.

Tenover, F. C., Arbeit, R. D., Goering, R. V., Mickelsen, P. A., Murray, B. E., Persing, D. H. \& Swaminathan, B. (1995). Interpreting chromoso- mal DNA restriction patterns produced by pulsed-field gel electrophoresis: criteria for bacterial strain typing. J Clin Microbiol 33, 22332239.

Yu, W. L., Pfaller, M. A., Winokur, P. L. \& Jones, R. N. (2002). Cefepime MIC as a predictor of the extended-spectrum $\beta$-lactamase type in Klebsiella pneumoniae, Taiwan. Emerg Infect Dis 8, 522-524. 